\title{
Riverine Autonomy
}

\author{
Daniel J. Stilwell \\ Bradley Department of Electrical and Computer Engineering \\ Virginia Tech \\ 340 Whittemore Hall \\ Blacksburg, VA 24061 \\ Phone: (540) 231-3204 Fax: (540) 231-3362Ｅmail: stilwell@vt.edu \\ Award Numbers: N00014-13-1-0411 \\ http://www.unmanned.vt.edu
}

\section{LONG-TERM GOALS}

The principal goal of this project is to develop the technology and algorithms that will enable an unmanned surface vehicle (USV) to operate fast and autonomously in unknown riverine environments, including tropical rivers. Robust autonomy requires that the USV senses the surface and subsurface environments, discriminates waterways that are navigable from those that are not, identifies stationary and moving obstacles, including other vessels, and then optimally plans and re-plans a route in realtime. Since speed is a vessel's principal defense, all of these tasks must be done as efficiently as possible to ensure successful operation at the greatest possible speed.

This project is tightly coordinated with collaborators at the Naval Postgraduate School (NPS) whose work is conducted under a related project.

\section{OBJECTIVES}

Specific objectives for VT and NPS during 2013 reported herein are

1. Development and testing of new SONAR mount for SOC-R to test sonar mounting concepts that resulted from prior experiments with NSW personnel.

2. Improvements to the Helmsman Assist System and preparation for experimental trials on SOC-R.

3. Improvements to the autonomy system so that overall mission risk is formally computed, so that plans are generated in real-time that minimize mission risk given whatever stochastic information about the environment.

4. Field trials of VT-USV autonomous operations in Peak Creek in Virginia, to thoroughly test the improved path planning algorithm for forward as well as backward path planning. 


\section{Report Documentation Page}

Form Approved

OMB No. 0704-0188

Public reporting burden for the collection of information is estimated to average 1 hour per response, including the time for reviewing instructions, searching existing data sources, gathering and maintaining the data needed, and completing and reviewing the collection of information. Send comments regarding this burden estimate or any other aspect of this collection of information,

including suggestions for reducing this burden, to Washington Headquarters Services, Directorate for Information Operations and Reports, 1215 Jefferson Davis Highway, Suite 1204, Arlington

VA 22202-4302. Respondents should be aware that notwithstanding any other provision of law, no person shall be subject to a penalty for failing to comply with a collection of information if it

does not display a currently valid OMB control number.

1. REPORT DATE

30 SEP 2013

4. TITLE AND SUBTITLE

Riverine Autonomy

6. $\operatorname{AUTHOR}(\mathrm{S})$

7. PERFORMING ORGANIZATION NAME(S) AND ADDRESS(ES)

Virginia Tech,Bradley Department of Electrical and Computer Engineering,340 Whittemore Hall,Blacksburg,VA,24061

9. SPONSORING/MONITORING AGENCY NAME(S) AND ADDRESS(ES)

3. DATES COVERED

00-00-2013 to 00-00-2013

5a. CONTRACT NUMBER

5b. GRANT NUMBER

5c. PROGRAM ELEMENT NUMBER

5d. PROJECT NUMBER

5e. TASK NUMBER

5f. WORK UNIT NUMBER

8. PERFORMING ORGANIZATION

REPORT NUMBER

10. SPONSOR/MONITOR'S ACRONYM(S)

11. SPONSOR/MONITOR'S REPORT

$\operatorname{NUMBER}(S)$

12. DISTRIBUTION/AVAILABILITY STATEMENT

Approved for public release; distribution unlimited

13. SUPPLEMENTARY NOTES

14. ABSTRACT

15. SUBJECT TERMS

16. SECURITY CLASSIFICATION OF:

a. REPORT

unclassified b. ABSTRACT

unclassified c. THIS PAGE

unclassified
17. LIMITATION OF ABSTRACT

Same as

Report (SAR)
18. NUMBER 19a. NAME OF

OF PAGES RESPONSIBLE PERSON

8 


\section{APPROACH}

We are developing technology and algorithm that enable a USV to autonomously travel over long distances in riverine environments. We are also developing the Helmsman Assist System as a first step toward integrating autonomy into riverine vessels. The Helmsman Assist System provides a display with real-time map and route-guidance from the Sensing and Autonomy Package. It enables manned vessels to operate much faster than would otherwise be possible in all weather and in unfamiliar areas where unknown submerged hazards may exist.

In September 2012, we demonstrated a preliminary version of the Sensing and Autonomy Package, along with the Helmsman Assist System, installed on the SOC-R. Based on the operational review by the NSWG4 operators, we began planning a new version of the sonar mounting system that will allow for rapid deployment as well as retraction. The new mounting system will also use a new sonar head to gather obstacle information along the vertical axis, thus improving subsea sensing performance.

For vehicle guidance, we seek to improve the path planning algorithm in three specific ways. First, we rigorously define the risk of travel in terms of probability of collision along the distance traveled by the USV. Apart from accounting for static obstacles, this approach allows the path planner to account for navigational hazards presented by dynamic obstacles such as floating debris. Second, we incorporate a dynamic model of the USV that accounts for effects of engine thrust and rudder on USV speed, turn rates, and accelerations. The path planner can thus generate smooth full state reference trajectories, instead of quasi-smooth paths defined by sequences of waypoints. By deploying a back-stepping controller developed previously, the USV can track reference trajectories at higher speeds. This approach also imparts maneuverability to the USV in cluttered environments and narrow waterways. Third, the path planner uses a-priori initial guesses of the optimal solution as inputs to the optimization algorithm. The optimal solution is computed by minimizing the probability of collision along the vehicle's path, while guaranteeing that the vehicle progresses towards the goal. Use of a-priori initial guesses considerably improves execution times, which is crucial to ensure safe and effective guidance at faster speeds.

\section{WORK COMPLETED}

\section{Sensing and Autonomy Package - New Sonar Mount for SOC-R}

Work has been completed on the design of a new generalized sonar mount. The new sonar mount incorporates many of the same design features of the previous mount deployed in FY12. It has automatic pan capability, tilt can be adjusted manually, a foam and fiberglass fairing is incorporated to reduce hydrodynamic drag on the pole, and nylon shear pins are designed to break and allow the pole to rotate freely if the sonar strikes an object or if the boat speed exceeds 10 knots.

Based on feedback from NSWG4 during field trials in FY12, several design requirements were developed for the new mount.

- The mount must not increase the effective beam width of the vessel.

- The mount should not interfere with normal operations onboard SOC-R.

- It should be capable of retracting quickly via a human-operated switch, or automatically when commanded by the Helmsman Assist System. 
- The mounting system must be temporary, and is therefore intended only for evaluating design choices of future permanent sonar mounting systems.

The new mount is designed to be positioned at the stern. The stern was chosen over the bow because it receives less traffic during deployment and extraction operations. It is also much closer to the water than the bow, which reduces the size and complexity of the sonar mount. A cradle is designed to mechanically interface with SOC-R's existing lift points. Special care was taken to ensure that the mount is low profile and does not interfere with the rear gunner. We also anticipate the potential for SOC-R's jets and hull to diminish sonar data quality, so the mount can position the sonar head roughly 2.5 feet below the hull. Deployment and retraction time for the new mount have been significantly improved from 30 seconds to an expected 3 seconds. These operations are achieved with a manual updown switch on the side of the electronics enclosure or via software commands.

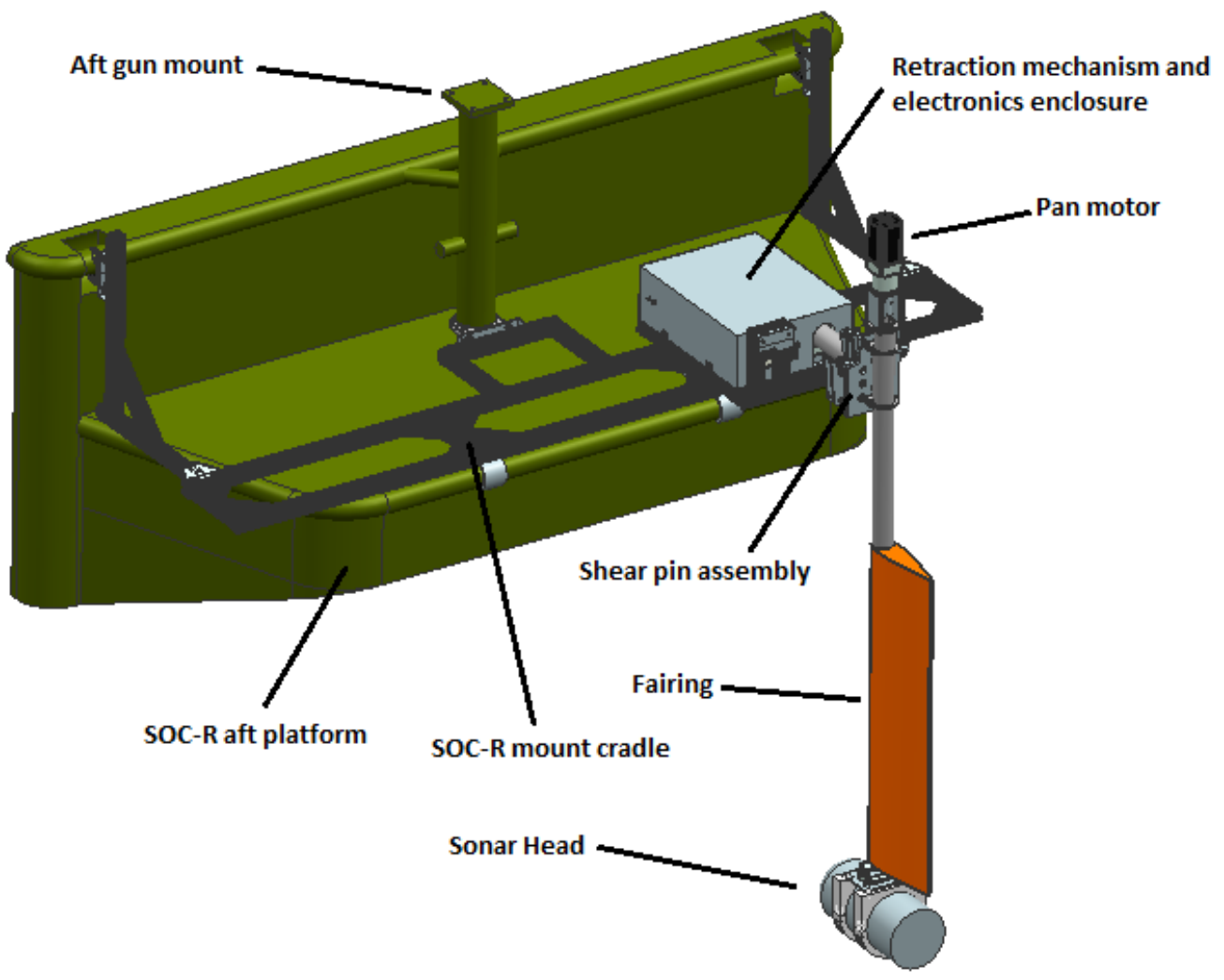

Figure 1: New sonar mount for SOC-R

\section{Improvements to the Path Planning Algorithm - Probabilistic formulation}

We have developed a formal framework that rigorously defines the risk of travel along a path in terms of probability of collision or other failure mode along that path. This allows the path planning algorithm to model the stochastic risk of collision not only near known obstacles, but also in open water channels where floating debris and other vehicular traffic may present dynamic navigation hazard. Given the map information in a local area and current state of global information, the path planner plans a path towards the goal that has the least probability of collision. 
The probability of collision along a distance $l$ can be expressed

$$
P_{c}=1-e^{-\alpha l}
$$

where, $\alpha$ can be thought of as the average number of failures per unit distance. We can also interpret $1 / \alpha$ to be the average distance the USV would travel before collision. Thus value of $\alpha$ is higher near obstacles and it decreases as distance to obstacle increases. We note that $\alpha$ can be rigorously computed from the map. The probability that USV will travel a distance $l$ without collision is

$$
P=e^{-\alpha l}
$$

Since value of $\alpha$ varies along the USV path, probability $P$ of not colliding along a path in a time interval $\left[t_{i}, t_{i}+T\right]$ is computed by parameterizing $\alpha$ and USV path $x$ as functions of time, and evaluating the line integral

$$
P\left(x\left(t_{i}\right)\right)=e^{-\int_{t_{i}}^{t_{i}+T} \alpha(x(t)) x^{l}(t) d t}
$$

The objective of the path planner is to plan paths that maximize this probability, and conversely minimize the probability of collision. The path planner generates a sequence of safe and dynamically feasible paths that guarantee convergence toward the goal. We solve the path planning problem by converting it into an optimal control problem, and employing the receding horizon framework over a finite interval $\left[t_{i}, t_{i}+T\right]$, to compute optimal control signals $u^{*}(t)$ and corresponding state trajectories $x^{*}(t)$ that satisfy

$$
u^{*}\left(t_{i}, x\left(t_{i}\right)\right)=\underset{u \in U}{\operatorname{argmin}} \int_{t_{i}}^{t_{i}+T} \alpha(x(t)) x^{\prime}(t) d t+W_{j}\left(x\left(t_{i}+T\right)\right)
$$

subject to the USV dynamics $\dot{x}(t)=f(x(t), u(t))$. The integral cost accounts for probability of collision within the planning horizon and the terminal cost $W_{j}\left(x\left(t_{i}+T\right)\right)$ accounts for global probability of collision from the end of the planning horizon up to the goal. The optimal control solution is then applied over an implementation horizon of length $\delta$.

We note that the terminal cost $W_{j}\left(x\left(t_{i}+T\right)\right)$ reflects global information that may be stale. In our approach, global information is updated infrequently and only when the so-called "matching condition" fails. The matching condition compares whether the risk of collision in the end section of length $\delta$, of the planned path, is smaller than the change in the global risk of collision within that section. If the risk is higher, then the matching condition fails and denotes a mismatch between current state of global information and local map information that has been obtained in real-time. A failed matching condition triggers a global update. Loosely speaking, the matching condition ensures that the direction of travel of the local path is generally similar to that of the globally safe passage near the end of the local planning horizon.

Figure 2 illustrates the use of matching condition during field trials in Peak Creek, VA. The short thick black line indicates the USV, the white line indicates local path planned and the black thin line indicates the globally safe passage. The top-left image in Figure 2 shows the case when the matching condition is satisfied and there is no mismatch between local and global information. As the USV travels, its onboard sensors identify previously unknown obstacles (top-right of Figure 2), thus introducing a mismatch between local and global information. That mismatch is not significant until the instant shown in top-right of Figure 2. Although it is not easily seen in the figure, the end part of the planned path diverges from the current globally safe passage. This triggers a global information update. The result of the global update is shown in the bottom-left of Figure 2, where the planned path 
and the globally safe passage have similar direction of travel. Results of field trials in Peak Creek are demonstrated in bottom-right of Figure 2, which shows the path taken by the USV to circumnavigate an unknown land-mass.
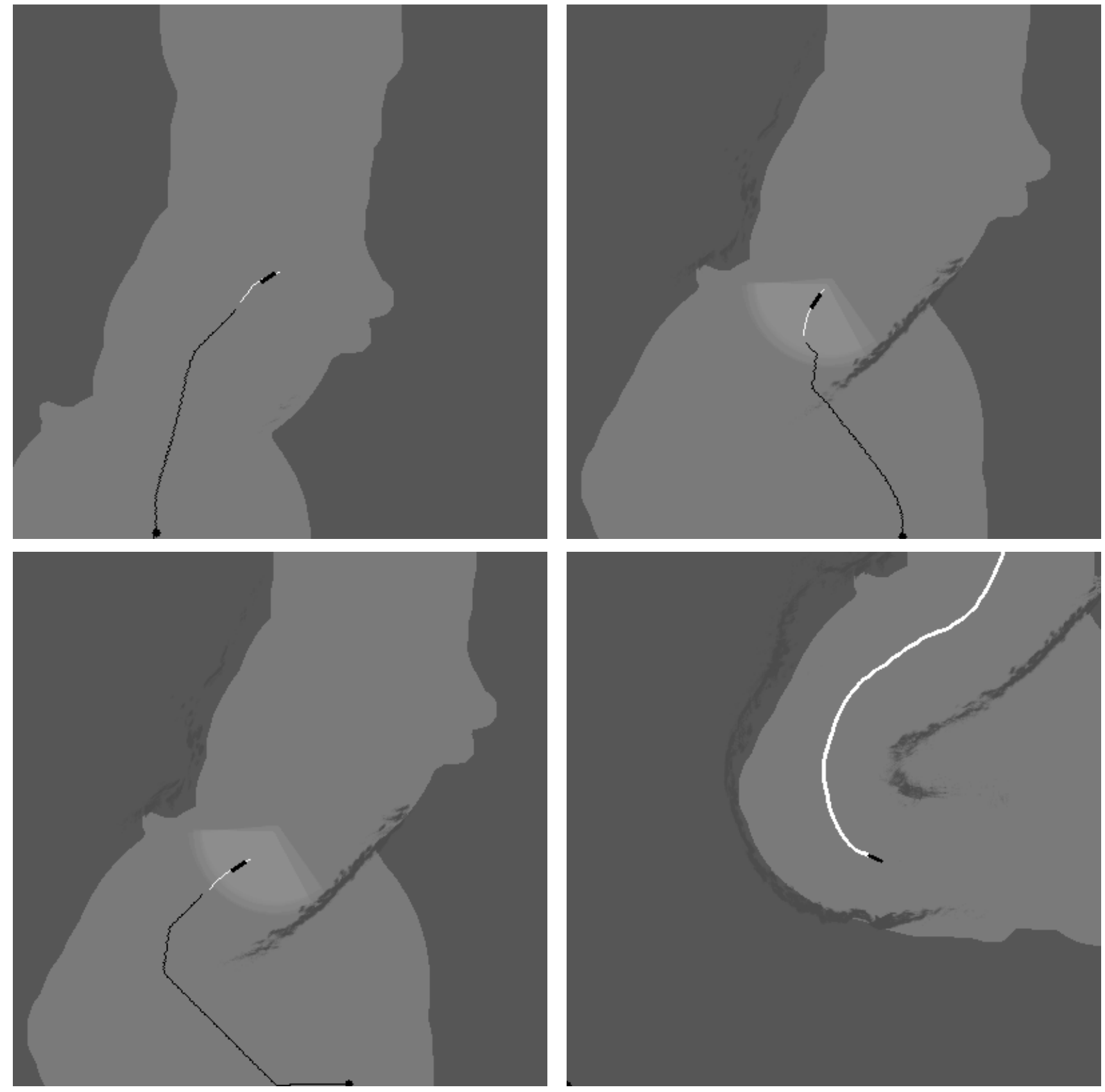

Figure 2: (top left) matching condition satisfied; (top right) matching condition violated; (bottom left) matching satisfied after global update; (bottom right) final USV path

\section{Improvements to the Path Planning Algorithm - USV dynamic model}

The path planner accounts for USV dynamics while solving the optimal control problem, guaranteeing that solution trajectories will be dynamically feasible for the USV. The dynamic model that was used 
earlier generated smooth trajectories; however it could produce piecewise continuous actuator commands. We have incorporated a full dynamic model of the USV that accounts for actuator dynamics, along with effects of engine thrust and rudder angle on USV speed, turn rates and accelerations. Thus not only the solution trajectories are smooth but the corresponding actuator commands are also smooth. The solution trajectories are tracked using a back-stepping controller. The use of full dynamic model for planning and back-stepping controller for tracking allows USV to navigate safely in cluttered environments and narrow waterways. It also enables the USV to operate at faster speeds.

\section{Improvements to the Path Planning Algorithm - Use of pre-computed initial guesses}

We implemented a new path planning strategy that is significantly different than that used earlier. The optimization algorithm converges to solution trajectory faster if a good initial guess is available. In the old implementation, an initial guess of optimal control actions was generated in real time using the Hybrid A* algorithm. The new strategy instead uses a number of predetermined initial guesses, thus reducing the computational requirements of the path planner during run-time. These initial guesses are not hard-coded in the software and can be easily changed through a text file, depending on the environment in which the USV is operating. The optimization algorithm uses all the available initial guesses to compute the solution. This strategy is especially beneficial in cluttered environments, where every initial guess seeds a solution which is close to locally optimal solution. The set of all such locally optimal trajectories are evaluated for probability of collision and the safest is chosen as the solution by the path planner. In an open area with very few obstacles, all initial guesses tend to collapse into a single solution. The left-hand side of Figure 3 shows the use of simple predetermined initial guesses when there are no obstacles in the vicinity of the USV. The blue square is the goal. The right-hand side of Figure 3 shows that all initial guesses collapse into very similar solutions. The path planner picks the solution with the least probability of collision. Thus, when USV is operating in open areas, a very small number of initial guesses can be sufficient.

Figure 4 shows initial guesses (left-hand side) and corresponding solutions (right-hand side) in a cluttered environment. In such a cluttered environment, some initial guesses may not produce a solution that is safe for navigation, and thus the planner needs a greater diversity of initial guesses. 

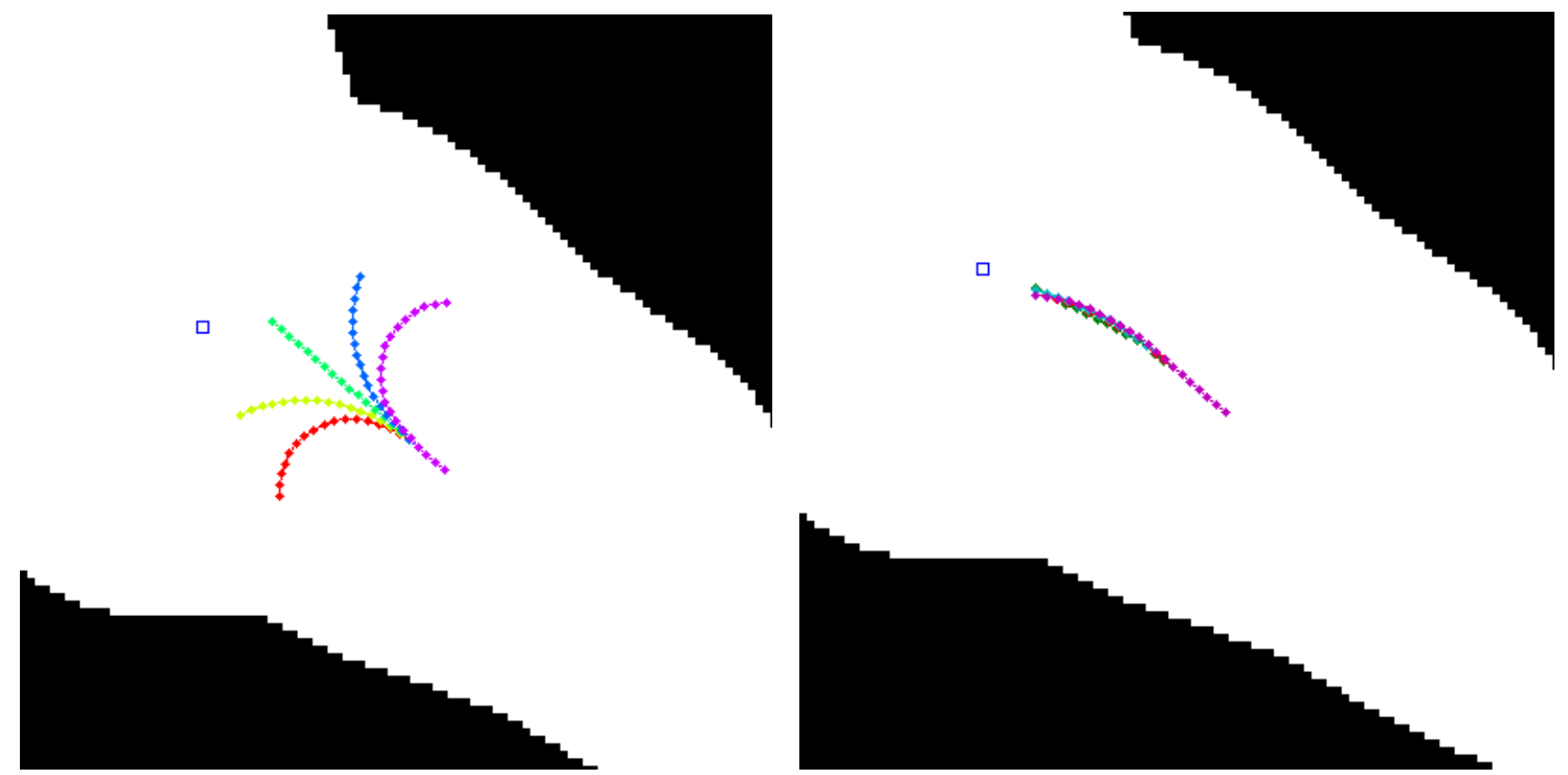

Figure 3: (left) Initial guesses without obstacles; (right) optimal solutions.

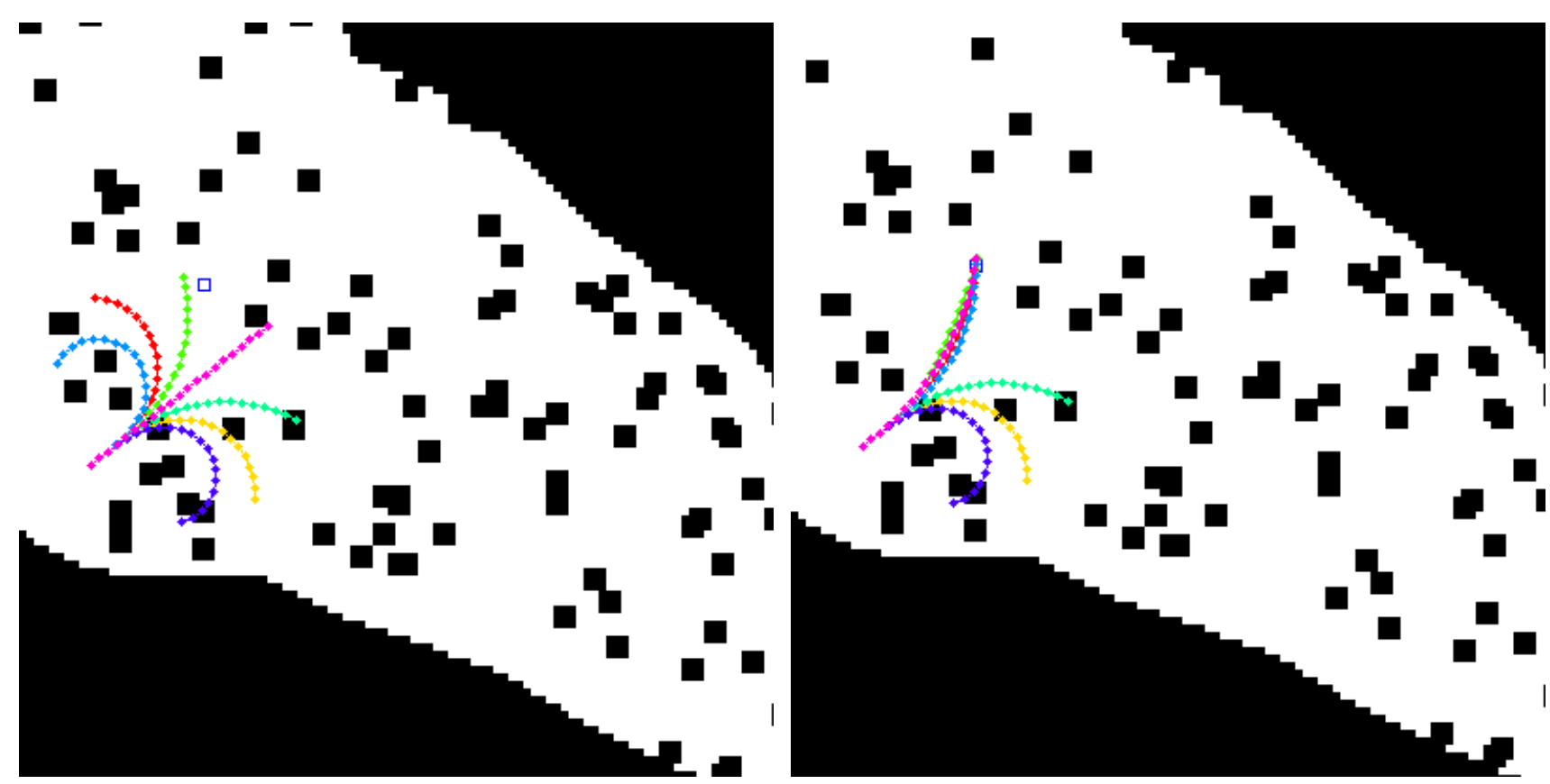

Figure 4: (left) Initial guesses near obstacles; (right) optimal solutions.

\section{IMPACT/APPLICATIONS}

The principal result of this project will be a set of algorithms and best-practice tools for robust autonomous surface vehicle operations in dynamic and partially mapped riverine systems. 


\section{RELATED PROJECTS}

None.

\section{PUBLICATIONS}

[1] B Xu, DJ Stilwell, AJ Kurdila, 2013, Fast path re-planning based on fast marching and level sets, Journal of Intelligent and Robotic Systems, 71:303-317.[peer-reviewed, published]

[2] AS Gadre, S Du, C Sonnenburg, D J Stilwell, CA Woolsey, 2012, Guidance and control of an unmanned surface vehicle exhibiting sternward motion, Proc. MTS/IEEE OCEANS, Hampton Roads, VA [peer-reviewed, published] 